\title{
The Complex Geometry of the Mandelbrot Set
}

\author{
Robert L. Devaney
}

\begin{abstract}
In this paper, we give a brief overview of the geometry of the Mandelbrot set. We show how to distinguish each of the principal bulbs hanging off the main cardioid of this set by counting the spokes of the antennas attached to each bulb. We also use these antennas to attach a fraction to each such bulb, and this then indicates how these bulbs are arranged around the boundary of the main cardioid.
\end{abstract}

Keywords Mandelbrot set - Julia set - Fatou set - Complex dynamics - Fractal • Farey addition $\cdot$ Quadratic dynamics

The Mandelbrot set $\mathcal{M}$ is one of the most interesting and beautiful objects in all of mathematics. It is also one of the most intricate planar sets. Contrary to the fact that it is named after Benoit Mandelbrot, the father of fractal geometry, this set is the antithesis of a fractal in that almost every tiny area of the boundary has its own "identity." That is, using some tools from geometry, we can read off exactly where this boundary point is and, more importantly, exactly what the corresponding dynamical behavior is.

Amazingly, the Mandelbrot set arises as the parameter plane for the seemingly simple quadratic family $P_{c}(z)=z^{2}+c$. See Fig. 1 . This is a picture in the $c$-plane (the parameter plane) that describes the fate of the orbit of the only critical point for this family, namely 0 . If the orbit of 0 does not tend to $\infty$, then the corresponding parameter $c$ lies in $\mathcal{M}$ and we color this point black. If the orbit does escape to $\infty$, then $c$ is not in $\mathcal{M}$ and we color $c$ according to how quickly the orbit of 0 reaches the exterior of a large disk surrounding the origin (with red points escaping fastest, followed in order by orange, yellow, green, blue, and violet).

2000 MSC number: Primary 37F10; Secondary 37F45

This work was partially supported by grant \#208780 from the Simons Foundation.

R. L. Devaney $(\varangle)$

Department of Mathematics, Boston University, 111 Cummington Mall,

Boston, MA 02215, USA

e-mail: bob@bu.edu 
Fig. 1 The Mandelbrot set. Colored points are $c$-values for which the orbits of 0 escape to $\infty$; black points are $c$-values for which this does not happen. So the Mandelbrot set is the black region in this image

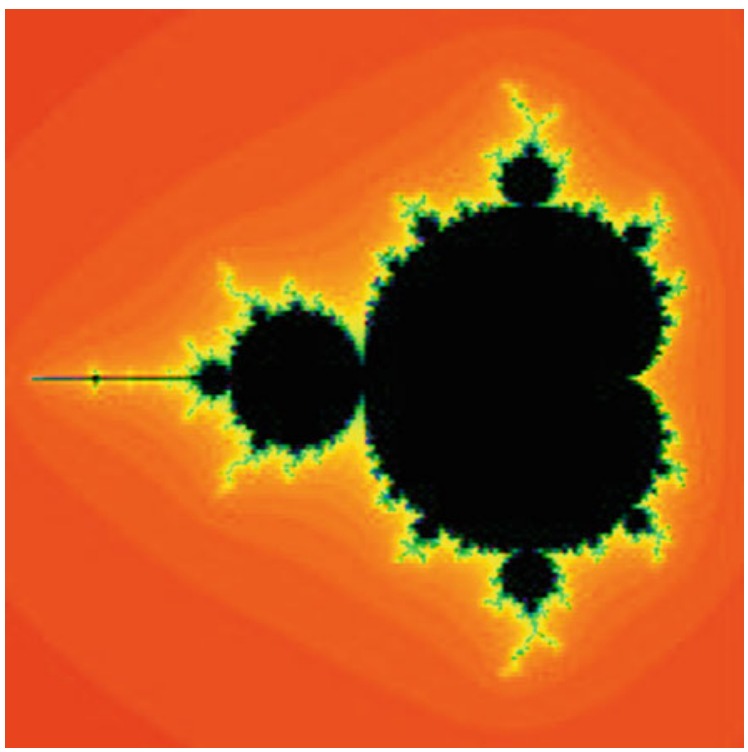

In complex dynamics, the object of central interest in the dynamical plane is the Julia set. For the family $P_{c}$, there is an open neighborhood of $\infty$ in the Riemann sphere consisting of points whose orbits tend to $\infty$. The set of all points whose orbits tend to $\infty$ is called the basin of $\infty$. Then the Julia set, denoted by $J\left(P_{c}\right)$ is the boundary of this basin. Given any point in the Julia set, then any open neighborhood of this point, no matter how small, is eventually mapped over the entire complex plane, minus at most one point. So the family of iterates of $P_{c}$ on the Julia set is very chaotic.

The natural question is: Why are we interested in the fate of the orbit of the critical point? Well, in short, the critical orbit "knows it all" in complex dynamics. In particular, for the family $P_{c}$, if the orbit of 0 tends to $\infty$, then the Julia set of $P_{c}$ is a Cantor set, i.e., a scatter of infinitely many points, or "fractal dust." If the orbit of 0 does not escape to $\infty$, then $J\left(P_{c}\right)$ is a connected set, i.e., just one piece.

The large black open regions (called hyperbolic components) visible in the Mandelbrot set are regions for which $P_{c}$ has an attracting cycle of some given period. It is known that, if $P_{c}$ has an attracting cycle, then the orbit of the critical point must tend to this cycle. Hence there can be at most one attracting cycle for a quadratic polynomial. For example, any $c$-value drawn from the central cardioid has an attracting fixed point. For $c$ in the large open disk just to the left of this cardioid, $P_{c}$ has an attracting 2-cycle. We therefore call this the period 2-bulb. And, for $c$ in the northernmost and southernmost bulbs off the main cardioid, $P_{c}$ has an attracting cycle of period 3, so these are the period 3-bulbs.

As $c$ moves from one hyperbolic component to another, the map undergoes a bifurcation. The simplest part of this bifurcation is the fact that we move from having 

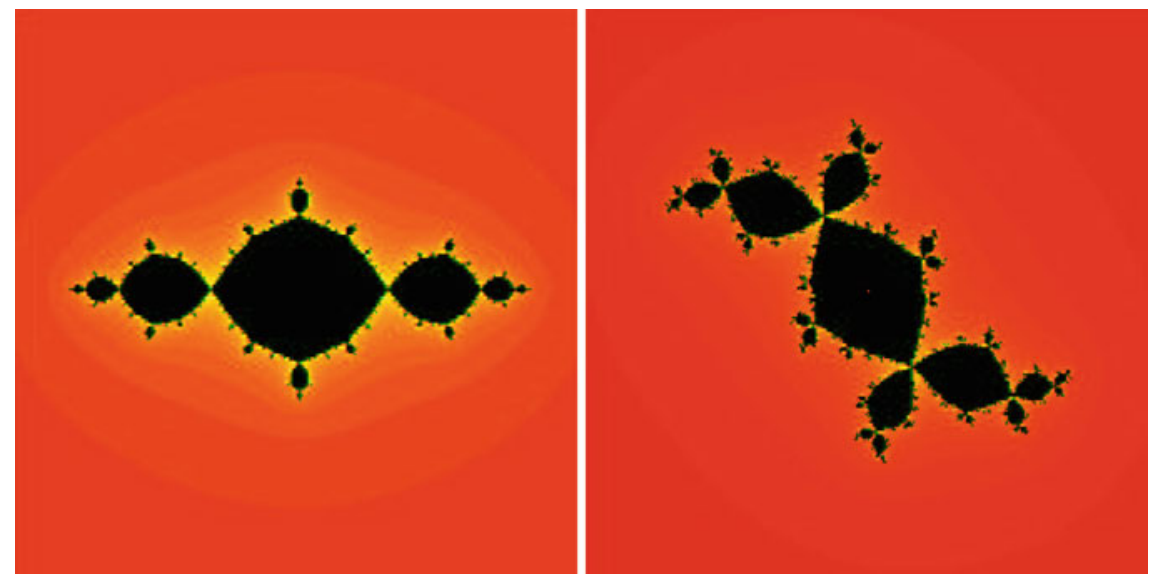

Fig. 2 The Julia sets for $z^{2}-1$ (the basilica) and $z^{2}-0.12+.75 i$ (the Douady rabbit). The filled Julia sets are the black regions, so the Julia sets here are the boundaries between the black and colored regions

an attracting cycle of some period when we are in one hyperbolic component to having an attracting cycle of some other period in the subsequent hyperbolic component. But, in fact, much more happens: the topology of the Julia sets changes dramatically. For example, if we move from the main cardioid to the period- 2 bulb, the Julia set, which is just a simple closed curve when $c$ is in the main cardioid, becomes a "basilica" when $c$ is in the period 2-bulb. What happens is a repelling 2-cycle that lies in $J\left(P_{c}\right)$ when $c$ is in the cardioid suddenly merges with the attracting fixed point and thereby makes it neutral when the parameter reaches the boundary of the cardioid. So two points in $J\left(P_{c}\right)$ become identified to one point. Meanwhile, infinitely many pairs of preimages of this point also become identified. This is what accounts for the infinitely many "pinch-points" visible in the basilica. Or, as we move from the main cardioid to the period 3-bulbs, a period 3-cycle becomes identified and the Julia set transforms into the "Douady rabbit". See Fig. 2.

A natural question is how do we understand how all of the bulbs and other smaller Mandelbrot sets are arranged in $\mathcal{M}$. Amazingly, if we zoom in to any portion of the boundary of the Mandelbrot set, it turns out that this zoom is very different from any other zoom that is non-symmetric with respect to $c \mapsto \bar{c}$. More importantly, with a keen eye for geometry, one can deduce exactly where in the boundary of $\mathcal{M}$ this zoom is, and, more importantly, what the corresponding dynamical behavior in the associated bulb is. It turns out that there are several different geometric and dynamical ways to understand the structure of these bulbs. We will look at this geometrically, but the real way to understand this uses techniques from complex analysis.

For simplicity, let's concentrate on the bulbs attached to the main cardioid. How do we know what their period is? One way is easy: look at the bulb. There is an antenna attached to this bulb. This antenna has a junction point from which a certain number of spokes emanate. The number of these spokes tells us exactly what the 

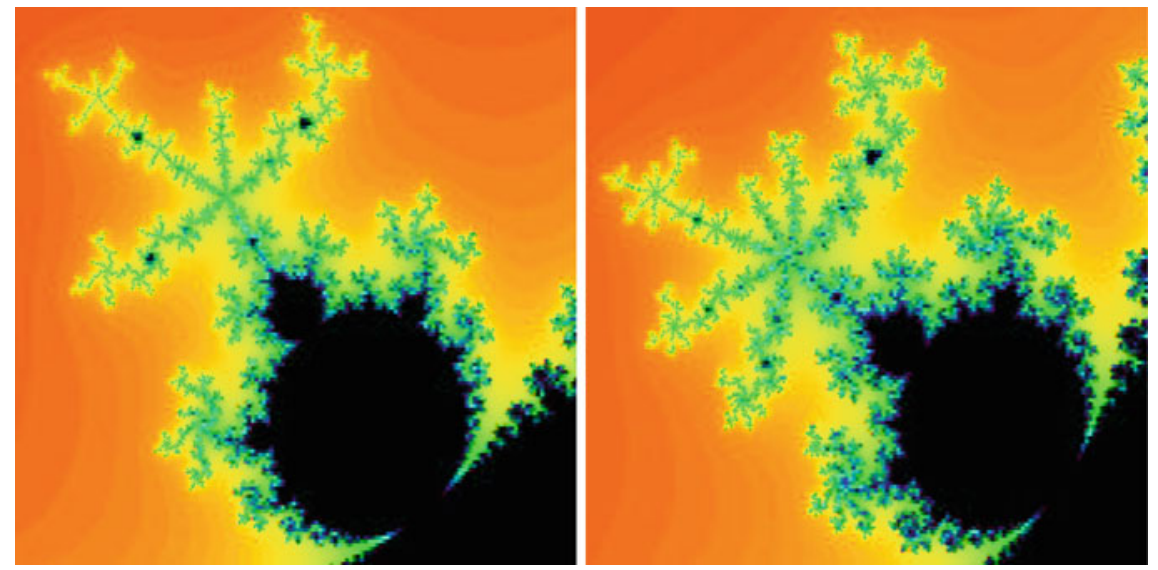

Fig. 3 Period 5 and 7 bulbs hanging off the main cardioid
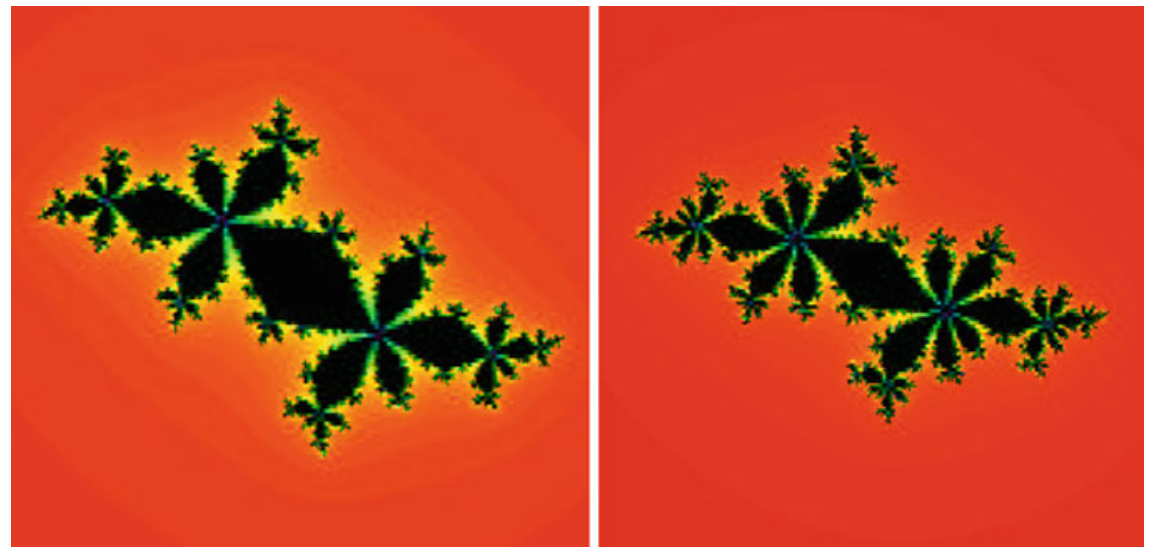

Fig. 4 Julia sets drawn from the above period 5 and 7 bulbs hanging off the main cardioid. Note that there are 4 and 6 "ears" hanging off the central disks of these filled Julia sets

period is. For example, in Fig. 3, we display two bulbs having periods 5 and 7 . Note that this is the exact number of antennas hanging off the junction point in the antenna of each bulb.

There is another way to read off the periods of these bulbs. Choose a parameter from the interior of a period $n$ bulb and plot the corresponding filled Julia set. There is a central disk in these filled Julia sets that surrounds the origin. Then there are exactly $n-1$ smaller disks that join this main disk at certain junction points. For example, in Fig. 2, we see that the rabbit has two "ears" attached to the central disk and the period of this bulb is $2+1=3$. Similarly, the basilica has just 1 ear and the period here is $1+1=2$. In Fig. 4, we display Julia sets from the above period 5 and period 7 bulbs, and we see the same phenomenon. 
Now let us turn to the arrangement of the bulbs around the main cardioid. To do this, we assign a fraction $p / q$ to each of these bulbs. Here $q$ is the period of the bulb, so the question is: what is $p$ ? There are several geometric and dynamical ways to determine $p$. Look at the period five bulb in Fig. 3. We call the spoke of the antenna that extends down to the bulb from the junction point the principal spoke. Note that the "shortest" spoke (that is not the principal spoke) is located 2/5 of a turn in the counter-clockwise direction from the principal spoke. And this bulb is then the 2/5-bulb. In that same figure, we also see that the period 7-bulb is, in fact, the 3/7-bulb.

A second way to see this is to turn to the filled Julia set. In Fig. 4, each of the filled Julia sets has a main component that surrounds the origin together with $q-1$ ears attached at one point. Note where the "smallest" ear is located; it is exactly $p / q$ of a turn in the counterclockwise direction from main component.

And then there is a third way to read off $p / q$. Simply plot the points on the attracting cycle of period $q$ in the Fatou set. What you see is that this cycle moves around the ears and the main component, rotating by $p / q$ of a turn at each stage. So there is a very nice connection between the geometry of the Mandelbrot set and Julia sets and the dynamics of $P_{c}$.

One curious fact that relates to the Farey tree involves the size of the bulbs hanging off the main cardioid. To begin, we think of the root point of the main cardioid as being the cusp at $c=1 / 4$. Then we call the main cardioid the $0 / 1$-bulb. The root point of any other bulb is just the point where this bulb is attached to the main cardioid. Now which is the largest bulb between the root points of the $0 / 1$ and $1 / 2$-bulbs (in, say, the upper portion of $\mathcal{M})$ ? It is clearly the $1 / 3$-bulb. And note that $1 / 3$ is obtained from the previous two fractions by Farey addition, i.e., adding the numerators and adding the denominators

$$
\frac{0}{1} "+\cdots \frac{1}{2}=\frac{1}{3} .
$$

Similarly, the largest bulb between the $1 / 3$ and $1 / 2$-bulbs is the $2 / 5$-bulb, again given by Farey addition.

$$
\frac{1}{3} ", " \frac{1}{2}=\frac{2}{5}
$$

And the largest bulb between the $2 / 5$ and 1/2-bulb is the $3 / 7$-bulb while the largest bulb between the $2 / 5$ and 1/3-bulbs is the 3/8-bulb and so on along the "Farey tree".

Then it follows that these bulbs are arranged around the boundary of the main cardioid in the exact order of the rational numbers in the unit interval. Actually, techniques from calculus can be used to prove this fairly easily. For more details, see [1-3]. An online, interactive discussion of this (with plenty of animations) called the Mandelbrot Set Explorer is available at http://math.bu.edu/DYSYS/explorer.

Using similar techniques from geometry, one can identify the other sub-bulbs in the Mandelbrot set. Unfortunately, there are many other points in the Mandelbrot set that this approach does not apply to; indeed, despite the simplicity of the function $z^{2}+c$, there are still many $c$-values in $\mathcal{M}$ for which we have no idea what is happening 
in the corresponding Julia set and what is the nearby structure in the Mandelbrot set. For example, along the boundary of the main cardioid we have only looked at the parameters corresponding to "rational" root points as discussed above. But there are uncountably many other points along the boundary of the cardioid. These correspond to "irrational" points. We understand the behavior of $P_{c}$ at the so-called "highly" irrational points, but the parameters at the "not-so-irrational" points have behavior that is still not understood. This is one of the major open problems in this area of mathematics. For a basic introduction to complex dynamics, see [4]. A more advanced survey of this field is John Milnor's book [5].

\section{References}

1. Devaney, R.L., Moreno Rocha, M.: Geometry of the antennas in the Mandelbrot set. Fractals 10, 39-46 (2002)

2. Devaney, R.L., Moreno Rocha, M.: The fractal geometry of the Mandelbrot set: I. periods of the bulbs. In: Fractals, Graphics, and Mathematics Education. MAA Notes, vol. 58, pp. 61-68 (2002)

3. Devaney, R.L., Moreno Rocha, M.: The fractal geometry of the Mandelbrot set: II. How to add and how to count. Fractals 3, 629-640 (1995)

4. Devaney, R.L.: An Introduction to Chaotic Dynamical Systems, 2nd edn. Westview Press, Boulder (2003)

5. Milnor, J.: Dynamics in One Complex Variable. Princeton University Press, Princeton (2006) 